\title{
Compactness of the set of trajectories of the control system described by a Urysohn
} type integral equation with quadratic integral constraints on the control functions

\author{
Idham A Alias ${ }^{1 *}$, Nesir Huseyin ${ }^{2}$ and Anar Huseyin ${ }^{3}$
}

\section{"Correspondence:}

idham_aa@upm.edu.my

'Department of Mathematics,

Faculty of Science, Universiti Putra

Malaysia, Serdang, Selangor 43400,

Malaysia

Full list of author information is

available at the end of the article

\begin{abstract}
In this paper the control system is considered described by a Urysohn type integral equation which is nonlinear with respect to the state vector and is affine with respect to the control vector. The functions from the space $L_{2}\left(\left[t_{0}, \theta\right] ; \mathbb{R}^{m}\right)$ satisfying a quadratic integral constraint are chosen as admissible control functions. The set of trajectories generated by all admissible control functions is studied. The boundedness, closedness, precompactness, and hence the compactness of the set of trajectories in the space of continuous functions is proved.
\end{abstract}

MSC: $45 \mathrm{G} 15 ; 93 \mathrm{C} 10$

Keywords: Urysohn type integral equation; control system; quadratic integral constraint; set of trajectories

\section{Introduction}

Different types of integral equations arise in various problems of theory and applications in mechanics, physics, biology, economics, medicine etc. (see, e.g. [1-12] and references therein). Many processes have exterior influences called control efforts or the system's disturbances. Therefore mathematical models of such processes include an additional parameter which is called the control or disturbance vector depending on the character of the exterior influences.

In the present paper, the control system described by a Urysohn type integral equation is studied. Note that the solution of the boundary value problem for an ordinary differential equation can be reduced to the solution of the suitable Urysohn type integral equation. Control systems described by a Urysohn type integral equation are considered in [1315], where it is assumed that the control functions satisfy the geometric constraint, which means that the control resource is not exhausted by consumption. But some kinds of control efforts are exhausted by consumption such as energy, fuel, finance, and food. In this case the integral constraint on the control functions is inevitable (see, e.g. [16-23] and references therein). For example, the mathematical model of the flying object with rapidly changing mass is described by a control system with integral constrained control functions (see, e.g. $[17,19,23])$. The various topological properties of the set of trajectories

(c) 2016 Alias et al. This article is distributed under the terms of the Creative Commons Attribution 4.0 International License (http://creativecommons.org/licenses/by/4.0/), which permits unrestricted use, distribution, and reproduction in any medium, provided you give appropriate credit to the original author(s) and the source, provide a link to the Creative Commons license, and indicate if changes were made. 
of the control systems described via an integral equation with integral constraint on the control functions are considered in [24-26].

Compactness of the set of trajectories of the control system described by a Urysohn type integral equation is investigated in this paper. It is assumed that the control functions are chosen from the space $L_{2}\left(\left[t_{0}, \theta\right] ; \mathbb{R}^{m}\right)$ and satisfy a quadratic integral constraint. Let us mention that compactness of the set of trajectories guaranties existence of the optimal trajectories in the optimal control problems with continuous payoff functionals.

The paper is organized as follows: In Section 2, the set of admissible control functions is defined and the boundedness, closedness, convexity, and weak compactness of the set of admissible control functions is shown (Proposition 2.2 and Proposition 2.3). In Section 3 the system and the basic conditions which satisfies the system is introduced (Conditions (3A), (3B), and (3C)). Existence and uniqueness of the system's trajectory generated by a given admissible control function are proved (Proposition 3.1). In Section 4 it is shown that the set of trajectories generated by all admissible control functions is a precompact subset of the space of continuous functions (Theorem 4.1). The closedness of the set of trajectories is proved in Section 5 (Proposition 5.1), and hence the compactness of the set of trajectories is obtained (Theorem 5.1).

\section{The set of admissible control functions}

We begin with the study of the set of admissible control functions. Let $Q(\cdot):\left[t_{0}, \theta\right] \rightarrow$ $\mathbb{R}^{m \times m}$ be a continuous matrix function and $Q(s)$ be a positive definite $m \times m$ matrix for every $s \in\left[t_{0}, \theta\right]$. The Lebesgue measurable function $u(\cdot) \in L_{2}\left(\left[t_{0}, \theta\right] ; \mathbb{R}^{m}\right)$ satisfying the inequality $\int_{t_{0}}^{\theta}\langle Q(s) u(s), u(s)\rangle d s \leq 1$ is said to be an admissible control function, where $L_{2}\left(\left[t_{0}, \theta\right] ; \mathbb{R}^{m}\right)$ is the space of Lebesgue measurable function $u(\cdot):\left[t_{0}, \theta\right] \rightarrow \mathbb{R}^{m}$ such that $\|u(\cdot)\|_{2}<+\infty$. Here $\|u(\cdot)\|_{2}=\left(\int_{t_{0}}^{\theta}\|u(t)\|^{2} d t\right)^{\frac{1}{2}},\|\cdot\|$ stands for the Euclidean norm, $\langle\cdot, \cdot\rangle$ denotes the scalar product. The set of all admissible control functions is denoted by the symbol $U$. Thus

$$
U=\left\{u(\cdot) \in L_{2}\left(\left[t_{0}, \theta\right] ; \mathbb{R}^{m}\right): \int_{t_{0}}^{\theta}\langle Q(s) u(s), u(s)\rangle d s \leq 1\right\} .
$$

Now let us give an auxiliary proposition which is used in the following arguments.

Proposition 2.1 Let $Q(\cdot):\left[t_{0}, \theta\right] \rightarrow \mathbb{R}^{m \times m}$ be a continuous matrix function and $Q(s)$ be a positive definite $m \times m$ matrix for every $s \in\left[t_{0}, \theta\right]$. Then there exist $c_{1}>0, c_{2}>0$ such that for each $u(\cdot) \in L_{2}\left(\left[t_{0}, \theta\right] ; \mathbb{R}^{m}\right)$ the inequality

$$
c_{1}^{2}\left(\|u(\cdot)\|_{2}\right)^{2} \leq \int_{t_{0}}^{\theta}\langle Q(s) u(s), u(s)\rangle d s \leq c_{2}^{2}\left(\|u(\cdot)\|_{2}\right)^{2}
$$

holds.

Proof Let $S_{m}=\left\{u \in \mathbb{R}^{m}:\|u\|=1\right\}$. For given $s \in\left[t_{0}, \theta\right]$ we set

$$
\gamma_{1}(s)=\min \left\{\langle Q(s) u, u\rangle: u \in S_{m}\right\}, \quad \gamma_{2}(s)=\max \left\{\langle Q(s) u, u\rangle: u \in S_{m}\right\} .
$$

Since the matrix function $Q(\cdot):\left[t_{0}, \theta\right] \rightarrow \mathbb{R}^{m \times m}$ is continuous and $Q(s)$ is a positive definite $m \times m$ matrix for every $s \in\left[t_{0}, \theta\right]$, the functions $\gamma_{1}(\cdot):\left[t_{0}, \theta\right] \rightarrow \mathbb{R}$ and $\gamma_{2}(\cdot):\left[t_{0}, \theta\right] \rightarrow \mathbb{R}$ are continuous, and $\gamma_{1}(s)>0, \gamma_{2}(s)>0$ for every $s \in\left[t_{0}, \theta\right]$. 
Now we denote

$$
\begin{aligned}
& c_{1}^{2}=\min \left\{\gamma_{1}(s): s \in\left[t_{0}, \theta\right]\right\}, \\
& c_{2}^{2}=\max \left\{\gamma_{2}(s): s \in\left[t_{0}, \theta\right]\right\} .
\end{aligned}
$$

It is obvious that $c_{1}>0$ and $c_{2}>0$. Thus for each $u \in S_{m}$ and $s \in\left[t_{0}, \theta\right]$, we have

$$
c_{1}^{2} \leq\langle Q(s) u, u\rangle \leq c_{2}^{2}
$$

Let us choose an arbitrary $u(\cdot) \in L_{2}\left(\left[t_{0}, \theta\right] ; \mathbb{R}^{m}\right)$. Then it follows from $(2.1)$ that

$$
c_{1}^{2} \leq\left\langle Q(s) \frac{u(s)}{\|u(s)\|}, \frac{u(s)}{\|u(s)\|}\right\rangle \leq c_{2}^{2}
$$

for every $s \in\left[t_{0}, \theta\right]$, where $u(s) \neq 0$. The last inequality implies the validity of the proposition.

From Proposition 2.1 follows the validity of the following corollary.

Corollary 2.1 For every $u(\cdot) \in U$ the inequality

$$
\|u(\cdot)\|_{2} \leq \frac{1}{c_{1}}
$$

is satisfied, where the number $c_{1}$ is defined in Proposition 2.1.

Let $u(\cdot) \in U$. Then from Hölder's inequality and Corollary 2.1 it follows that the inequality

$$
\int_{t_{0}}^{\theta}\|u(s)\| d s \leq \frac{1}{c_{1}} \sqrt{\theta-t_{0}}
$$

is verified.

Proposition 2.2 The set of admissible control functions $U$ is a bounded, closed, and convex subset of the space $L_{2}\left(\left[t_{0}, \theta\right] ; \mathbb{R}^{m}\right)$.

Proof The boundedness of the set of admissible control functions $U$ follows from Corollary 2.1.

Let us show closedness of the set $U$. Assume that $u_{k}(\cdot) \in U$ for $k=1,2, \ldots$ and $\| u_{k}(\cdot)-$ $u_{*}(\cdot) \|_{2} \rightarrow 0$ as $k \rightarrow \infty$. We will show that $u_{*}(\cdot) \in U$, i.e., $\int_{t_{0}}^{\theta}\left\langle Q(s) u_{*}(s), u_{*}(s)\right\rangle d s \leq 1$.

It is not difficult to verify that

$$
\begin{gathered}
\left|\int_{t_{0}}^{\theta}\left\langle Q(s) u_{k}(s), u_{k}(s)\right\rangle d s-\int_{t_{0}}^{\theta}\left\langle Q(s) u_{*}(s), u_{*}(s)\right\rangle d s\right| \\
\leq \int_{t_{0}}^{\theta}\|Q(s)\|\left\|u_{k}(s)\right\|\left\|u_{k}(s)-u_{*}(s)\right\| d s \\
\quad+\int_{t_{0}}^{\theta}\|Q(s)\|\left\|u_{*}(s)\right\|\left\|u_{k}(s)-u_{*}(s)\right\| d s
\end{gathered}
$$


for every $k=1,2, \ldots$. Since the function $Q(\cdot):\left[t_{0}, \theta\right] \rightarrow \mathbb{R}^{m \times m}$ is continuous, there exists $a_{*}>0$ such that $\|Q(s)\| \leq a_{*}$ for every $s \in\left[t_{0}, \theta\right]$. Then (2.3) and Hölder's inequality imply that

$$
\begin{aligned}
& \left|\int_{t_{0}}^{\theta}\left\langle Q(s) u_{k}(s), u_{k}(s)\right\rangle d s-\int_{t_{0}}^{\theta}\left\langle Q(s) u_{*}(s), u_{*}(s)\right\rangle d s\right| \\
& \quad \leq a_{*}\left\|u_{k}(\cdot)\right\|_{2} \cdot\left\|u_{k}(\cdot)-u_{*}(\cdot)\right\|_{2}+a_{*}\left\|u_{*}(\cdot)\right\|_{2} \cdot\left\|u_{k}(\cdot)-u_{*}(\cdot)\right\|_{2}
\end{aligned}
$$

for every $k=1,2, \ldots$ Since $\left\|u_{k}(\cdot)-u_{*}(\cdot)\right\|_{2} \rightarrow 0$ as $k \rightarrow \infty$, there exists $a_{1}>0$ such that $\left\|u_{*}(\cdot)\right\|_{2} \leq a_{1},\left\|u_{k}(\cdot)\right\|_{2} \leq a_{1}$ for every $k=1,2, \ldots$. Thus the last inequality yields

$$
\left|\int_{t_{0}}^{\theta}\left\langle Q(s) u_{k}(s), u_{k}(s)\right\rangle d s-\int_{t_{0}}^{\theta}\left\langle Q(s) u_{*}(s), u_{*}(s)\right\rangle d s\right| \leq 2 a_{*} a_{1}\left\|u_{k}(\cdot)-u_{*}(\cdot)\right\|_{2}
$$

for every $k=1,2, \ldots$. The inclusions $u_{k}(\cdot) \in U, k=1,2, \ldots$, imply that

$$
\int_{t_{0}}^{\theta}\left\langle Q(s) u_{k}(s), u_{k}(s)\right\rangle d s \leq 1
$$

for every $k=1,2, \ldots$ From (2.4) and (2.5) we obtain

$$
\begin{aligned}
\int_{t_{0}}^{\theta}\left\langle Q(s) u_{*}(s), u_{*}(s)\right\rangle d s & \leq \int_{t_{0}}^{\theta}\left\langle Q(s) u_{k}(s), u_{k}(s)\right\rangle d s+2 a_{*} a_{1}\left\|u_{k}(\cdot)-u_{*}(\cdot)\right\|_{2} \\
& \leq 1+2 a_{*} a_{1}\left\|u_{k}(\cdot)-u_{*}(\cdot)\right\|_{2}
\end{aligned}
$$

for every $k=1,2, \ldots$ and hence

$$
\int_{t_{0}}^{\theta}\left\langle Q(s) u_{*}(s), u_{*}(s)\right\rangle d s \leq 1
$$

Thus $u_{*}(\cdot) \in U$.

Now, let us show the convexity of the set $U$.

Since the matrix $Q(s)$ is positive definite for every $s \in\left[t_{0}, \theta\right]$, then it is possible to specify that the function $u \rightarrow\langle Q(s) u, u\rangle, u \in \mathbb{R}^{m}$, is convex for every $s \in\left[t_{0}, \theta\right]$ (see [27]).

Let $u_{1}(\cdot) \in U, u_{2}(\cdot) \in U$, and $\alpha \in[0,1]$. Then from the convexity of the function $u \rightarrow$ $\langle Q(s) u, u\rangle, u \in \mathbb{R}^{m}$, for every $s \in\left[t_{0}, \theta\right]$ it follows that

$$
\begin{gathered}
\left\langle Q(s)\left(\alpha u_{1}(s)+(1-\alpha) u_{2}(s)\right),\left(\alpha u_{1}(s)+(1-\alpha) u_{2}(s)\right)\right\rangle \\
\leq \alpha\left\langle Q(s) u_{1}(s), u_{1}(s)\right\rangle+(1-\alpha)\left\langle Q(s) u_{2}(s), u_{2}(s)\right\rangle
\end{gathered}
$$

for every $s \in\left[t_{0}, \theta\right]$, and consequently

$$
\begin{aligned}
& \int_{t_{0}}^{\theta}\left\langle Q(s)\left(\alpha u_{1}(s)+(1-\alpha) u_{2}(s)\right),\left(\alpha u_{1}(s)+(1-\alpha) u_{2}(s)\right)\right\rangle d s \\
& \quad \leq \alpha \int_{t_{0}}^{\theta}\left\langle Q(s) u_{1}(s), u_{1}(s)\right\rangle d s+(1-\alpha) \int_{t_{0}}^{\theta}\left\langle Q(s) u_{2}(s), u_{2}(s)\right\rangle d s \\
& \quad \leq \alpha+(1-\alpha)=1 .
\end{aligned}
$$

This means that $\alpha u_{1}(\cdot)+(1-\alpha) u_{2}(\cdot) \in U$ and the proof is completed. 
Proposition 2.3 The set of admissible control functions $U$ is a weakly compact subset of the space $L_{2}\left(\left[t_{0}, \theta\right], \mathbb{R}^{m}\right)$.

Proof Let $u_{k}(\cdot) \in U$ for every $k=1,2, \ldots$. Let us show that there exists a subsequence $\left\{u_{k_{i}}(\cdot)\right\}_{i=1}^{\infty}$ of the sequence $\left\{u_{k}(\cdot)\right\}_{k=1}^{\infty}$ and $u_{*}(\cdot) \in U$ such that $u_{k_{i}}(\cdot) \stackrel{\text { weak }}{\longrightarrow} u_{*}(\cdot)$ as $i \rightarrow \infty$.

Since $u_{k}(\cdot) \in U$ for every $k=1,2, \ldots$, by virtue of the Corollary 2.1 we see that the sequence $\left\{u_{k}(\cdot)\right\}_{k=1}^{\infty}$ is bounded in the space $L_{2}\left(\left[t_{0}, \theta\right], \mathbb{R}^{m}\right)$, and hence according to [28] it has a weakly convergent subsequence $\left\{u_{k_{i}}(\cdot)\right\}_{i=1}^{\infty}$. Let $u_{k_{i}}(\cdot) \stackrel{\text { weak }}{\longrightarrow} u_{*}(\cdot)$ as $i \rightarrow \infty$.

By Mazur's theorem (see, e.g. [29]), for each $j>0$, there exist $\alpha_{1}^{j} \geq 0, \alpha_{2}^{j} \geq 0, \ldots, \alpha_{j}^{j} \geq 0$ such that $\sum_{i=1}^{j} \alpha_{i}^{j}=1$ and

$$
\left\|\sum_{i=1}^{j} \alpha_{i}^{j} u_{k_{i}}(\cdot)-u_{*}(\cdot)\right\|_{2}<\frac{1}{j} .
$$

Let us denote $z_{j}(\cdot)=\sum_{i=1}^{j} \alpha_{i}^{j} u_{k_{i}}(\cdot)$. Since $\alpha_{1}^{j} \geq 0, \alpha_{2}^{j} \geq 0, \ldots, \alpha_{j}^{j} \geq 0, \sum_{i=1}^{j} \alpha_{i}^{j}=1, u_{k_{i}}(\cdot) \in U$ for every $i=1,2, \ldots$ and $U \subset L_{2}\left(\left[t_{0}, \theta\right], \mathbb{R}^{m}\right)$ is a convex set (according to the Proposition 2.2), we have $z_{j}(\cdot) \in U$ for every $j=1,2, \ldots$. Thus, from (2.6) we conclude that for a given $j>0$ there exists $z_{j}(\cdot) \in U$ such that the inequality

$$
\left\|z_{j}(\cdot)-u_{*}(\cdot)\right\|_{2}<\frac{1}{j}
$$

holds. This means that $u_{*}(\cdot) \in \operatorname{cl}(U)$, where $c l$ denotes the closure of a set. Via Proposition 2.2, $U$ is a closed set. Then we obtain $u_{*}(\cdot) \in U$.

\section{The system and the set of trajectories}

We consider a control system the behavior of which is described by a Urysohn type integral equation

$$
x(t)=f(t, x(t))+\lambda \int_{t_{0}}^{\theta}\left[K_{1}(t, s, x(s))+K_{2}(t, s, x(s)) u(s)\right] d s,
$$

where $t \in\left[t_{0}, \theta\right], s \in\left[t_{0}, \theta\right], x(s) \in \mathbb{R}^{n}$ is the state vector, $u(s) \in \mathbb{R}^{m}$ is the control vector and $\lambda \geq 0$.

We assume that the functions and the number $\lambda \geq 0$ given in system (3.1) satisfy the following conditions:

(3A) the functions $f(\cdot):\left[t_{0}, \theta\right] \times \mathbb{R}^{n} \rightarrow \mathbb{R}^{n}, K_{1}(\cdot):\left[t_{0}, \theta\right] \times\left[t_{0}, \theta\right] \times \mathbb{R}^{n} \rightarrow \mathbb{R}^{n}$, and $K_{2}(\cdot):\left[t_{0}, \theta\right] \times\left[t_{0}, \theta\right] \times \mathbb{R}^{n} \rightarrow \mathbb{R}^{n \times m}$ are continuous;

(3B) there exist $L_{0} \in[0,1), L_{1} \geq 0$, and $L_{2} \geq 0$ such that

$$
\begin{aligned}
& \left\|f\left(t, x_{1}\right)-f\left(t, x_{2}\right)\right\| \leq L_{0}\left\|x_{1}-x_{2}\right\|, \\
& \left\|K_{1}\left(t, s, x_{1}\right)-K_{1}\left(t, s, x_{2}\right)\right\| \leq L_{1}\left\|x_{1}-x_{2}\right\|, \\
& \left\|K_{2}\left(t, s, x_{1}\right)-K_{2}\left(t, s, x_{2}\right)\right\| \leq L_{2}\left\|x_{1}-x_{2}\right\|
\end{aligned}
$$

for every $\left(t, x_{1}\right) \in\left[t_{0}, \theta\right] \times \mathbb{R}^{n},\left(t, x_{2}\right) \in\left[t_{0}, \theta\right] \times \mathbb{R}^{n},\left(t, s, x_{1}\right) \in\left[t_{0}, \theta\right] \times\left[t_{0}, \theta\right] \times \mathbb{R}^{n}$, $\left(t, s, x_{2}\right) \in\left[t_{0}, \theta\right] \times\left[t_{0}, \theta\right] \times \mathbb{R}^{n} ;$ 
(3C) the inequality $\lambda L_{1}\left(\theta-t_{0}\right)+\lambda L_{2} \sqrt{\theta-t_{0}} \frac{1}{c_{1}}<1-L_{0}$ is satisfied, where $c_{1}$ is defined in Proposition 2.1.

We denote

$$
L(\lambda)=L_{0}+\lambda L_{1}\left(\theta-t_{0}\right)+\lambda L_{2} \sqrt{\theta-t_{0}} \frac{1}{c_{1}} .
$$

By virtue of condition (3C) we have $L(\lambda)<1$.

Now, let us define the trajectory of the system (3.1) generated by a given admissible control function. Let $u_{*}(\cdot) \in U$. A continuous function $x_{*}(\cdot):\left[t_{0}, \theta\right] \rightarrow \mathbb{R}^{n}$ satisfying the integral equation

$$
x_{*}(t)=f\left(t, x_{*}(t)\right)+\lambda \int_{t_{0}}^{\theta}\left[K_{1}\left(t, s, x_{*}(s)\right)+K_{2}\left(t, s, x_{*}(s)\right) u_{*}(s)\right] d s
$$

for each $t \in\left[t_{0}, \theta\right]$ is called a trajectory of the system (3.1), generated by the admissible control function $u_{*}(\cdot) \in U$.

The trajectory of the system (3.1) generated by the control function $u(\cdot) \in U$ is denoted by $x(\cdot ; u(\cdot))$ and the set

$$
\mathbf{X}=\{x(\cdot ; u(\cdot)): u(\cdot) \in U\}
$$

is called the set of trajectories of the system (3.1). It is obvious that $\mathbf{X} \subset C\left(\left[t_{0}, \theta\right] ; \mathbb{R}^{n}\right)$, where $C\left(\left[t_{0}, \theta\right] ; \mathbb{R}^{n}\right)$ is the space of continuous functions $x(\cdot):\left[t_{0}, \theta\right] \rightarrow \mathbb{R}^{n}$ with norm

$$
\|x(\cdot)\|_{C}=\max \left\{\|x(t)\|: t \in\left[t_{0}, \theta\right]\right\} .
$$

For $t \in\left[t_{0}, \theta\right]$ we denote

$$
\mathbf{X}(t)=\left\{x(t) \in \mathbb{R}^{n}: x(\cdot) \in \mathbf{X}\right\}
$$

The set $\mathbf{X}(t)$ consists of points to which arrive the trajectories of the system at the instant of $t$.

Proposition 3.1 Every $u(\cdot) \in U$ generates a unique trajectory of the system (3.1).

Proof Let $u_{*}(\cdot) \in U$ be a fixed admissible control function. Define an operator $F(x(\cdot))$ by setting

$$
F(x(\cdot)) \mid(t)=f(t, x(t))+\lambda \int_{t_{0}}^{\theta}\left[K_{1}(t, s, x(s))+K_{2}(t, s, x(s)) u_{*}(s)\right] d s, \quad t \in\left[t_{0}, \theta\right]
$$

where $x(\cdot) \in C\left(\left[t_{0}, \theta\right], \mathbb{R}^{n}\right)$.

It is not difficult to prove that, for each fixed $x(\cdot) \in C\left(\left[t_{0}, \theta\right] ; \mathbb{R}^{n}\right)$, the function $t \mapsto$ $F(x(\cdot)) \mid(t), t \in\left[t_{0}, \theta\right]$, is continuous. So is the operator

$$
F(\cdot): C\left(\left[t_{0}, \theta\right] ; \mathbb{R}^{n}\right) \rightarrow C\left(\left[t_{0}, \theta\right] ; \mathbb{R}^{n}\right) .
$$


Let us choose arbitrarily $x_{1}(\cdot) \in C\left(\left[t_{0}, \theta\right] ; \mathbb{R}^{n}\right), x_{2}(\cdot) \in C\left(\left[t_{0}, \theta\right] ; \mathbb{R}^{n}\right)$ and $t \in\left[t_{0}, \theta\right]$. Then from condition (3B) and (2.2) it follows that

$$
\begin{aligned}
&\left\|F\left(x_{2}(\cdot)\right)\left|(t)-F\left(x_{1}(\cdot)\right)\right|(t)\right\| \\
& \leq L_{0}\left\|x_{2}(t)-x_{1}(t)\right\|+\lambda L_{1} \int_{t_{0}}^{\theta}\left\|x_{2}(s)-x_{1}(s)\right\| d s \\
& \quad+\lambda L_{2} \int_{t_{0}}^{\theta}\left\|x_{2}(s)-x_{1}(s)\right\|\left\|u_{*}(s)\right\| d s \\
& \leq\left[L_{0}+\lambda L_{1}\left(\theta-t_{0}\right)+\lambda L_{2} \int_{t_{0}}^{\theta}\left\|u_{*}(s)\right\| d s\right]\left\|x_{2}(\cdot)-x_{1}(\cdot)\right\|_{C} \\
& \leq {\left[L_{0}+\lambda L_{1}\left(\theta-t_{0}\right)+\lambda L_{2} \sqrt{\theta-t_{0}} \cdot \frac{1}{c_{1}}\right]\left\|x_{2}(\cdot)-x_{1}(\cdot)\right\|_{C} }
\end{aligned}
$$

for every $t \in\left[t_{0}, \theta\right]$, and hence

$$
\left\|F\left(x_{2}(\cdot)\right)-F\left(x_{1}(\cdot)\right)\right\|_{C} \leq L(\lambda)\left\|x_{2}(\cdot)-x_{1}(\cdot)\right\|_{C}
$$

where $L(\lambda)$ is defined by (3.2).

Via Condition (3C) we have $L(\lambda)<1$. Then (3.4) shows that the operator $F(\cdot): C\left(\left[t_{0}, \theta\right]\right.$; $\left.\mathbb{R}^{n}\right) \rightarrow C\left(\left[t_{0}, \theta\right] ; \mathbb{R}^{n}\right)$ is contractive. Since $C\left(\left[t_{0}, \theta\right], \mathbb{R}^{n}\right)$ is a complete metric space, by the Banach fixed point theorem, the operator $F(\cdot)$ has a unique fixed point, that is, there exists a unique $x_{*}(\cdot) \in C\left(\left[t_{0}, \theta\right] ; \mathbb{R}^{n}\right)$ such that $F\left(x_{*}(\cdot)\right)=x_{*}(\cdot)$, which means that there exists a unique $x_{*}(\cdot) \in C\left(\left[t_{0}, \theta\right] ; \mathbb{R}^{n}\right)$ such that

$$
x_{*}(t)=f\left(t, x_{*}(t)\right)+\lambda \int_{a}^{b}\left[K_{1}\left(t, s, x_{*}(s)\right)+K_{2}\left(t, s, x_{*}(s)\right) u_{*}(s)\right] d s
$$

for every $\left[t_{0}, \theta\right]$.

\section{Precompactness of the set of trajectories}

First of all we will prove that the set of trajectories $\mathbf{X}$ of the system (3.1) is a bounded subset of the space $C\left(\left[t_{0}, \theta\right] ; \mathbb{R}^{n}\right)$.

Proposition 4.1 There exist $\beta_{0} \geq 0, \beta_{1} \geq 0, \beta_{2} \geq 0$ such that

(i) $\|f(t, x)\| \leq \beta_{0}+L_{0}\|x\|$,

(ii) $\left\|K_{1}(t, s, x)\right\| \leq \beta_{1}+L_{1}\|x\|$,

(iii) $\left\|K_{2}(t, s, x)\right\| \leq \beta_{2}+L_{2}\|x\|$,

for every $(t, x) \in\left[t_{0}, \theta\right] \times \mathbb{R}^{n}$ and $(t, s, x) \in\left[t_{0}, \theta\right] \times\left[t_{0}, \theta\right] \times \mathbb{R}^{n}$, where $L_{0}, L_{1}$, and $L_{2}$ are defined in Condition (3B).

Proof We just show the proof for (iii). According to Condition (3B)

$$
\left\|K_{2}(t, s, x)-K_{2}(t, s, 0)\right\| \leq L_{2}\|x\|
$$

for every $(t, s, x) \in\left[t_{0}, \theta\right] \times\left[t_{0}, \theta\right] \times \mathbb{R}^{n}$ and consequently,

$$
\left\|K_{2}(t, s, x)\right\| \leq\left\|K_{2}(t, s, 0)\right\|+L_{2}\|x\|
$$


for every $(t, s, x) \in\left[t_{0}, \theta\right] \times\left[t_{0}, \theta\right] \times \mathbb{R}^{n}$. Since the function $K_{2}(\cdot)$ is continuous, setting

$$
\beta_{2}=\max \left\{\left\|K_{2}(t, s, 0)\right\|:(t, s) \in\left[t_{0}, \theta\right] \times\left[t_{0}, \theta\right]\right\}
$$

we obtain the proof of (iii) from (4.1). The proofs of (i) and (ii) are carried out similarly.

\section{Denote}

$$
q_{*}=\frac{\beta_{0}+\lambda \beta_{1}\left(\theta-t_{0}\right)+\lambda \beta_{2} \sqrt{\theta-t_{0}} \frac{1}{c_{1}}}{1-L(\lambda)}
$$

where $L(\lambda)$ is defined by (3.2).

Proposition 4.2 For every $x(\cdot) \in \mathbf{X}$ the inequality

$$
\|x(\cdot)\|_{C} \leq q_{*}
$$

is satisfied.

Proof Let $x(\cdot) \in \mathbf{X}$ be an arbitrary chosen trajectory, generated by the control function $u(\cdot) \in U$. Then

$$
x(t)=f(t, x(t))+\lambda \int_{t_{0}}^{\theta}\left[K_{1}(t, s, x(s))+K_{2}(t, s, x(s)) u(s)\right] d s
$$

for each $t \in\left[t_{0}, \theta\right]$. Proposition 4.1, (2.2), and (3.2) imply that

$$
\begin{aligned}
\|x(t)\| \leq & \beta_{0}+L_{0}\|x(t)\|+\lambda \int_{t_{0}}^{\theta}\left[\left(\beta_{1}+L_{1}\|x(s)\|\right)+\left(\beta_{2}+L_{2}\|x(s)\|\right)\|u(s)\|\right] d s \\
\leq & \beta_{0}+L_{0}\|x(\cdot)\|_{C}+\lambda \beta_{1}\left(\theta-t_{0}\right)+\lambda L_{1}\|x(\cdot)\|_{C}\left(\theta-t_{0}\right) \\
& +\lambda \beta_{2} \int_{t_{0}}^{\theta}\|u(s)\| d s+\lambda L_{2}\|x(\cdot)\|_{C} \int_{t_{0}}^{\theta}\|u(s)\| d s \\
\leq & \beta_{0}+\lambda \beta_{1}\left(\theta-t_{0}\right)+\lambda \beta_{2} \sqrt{\theta-t_{0}} \frac{1}{c_{1}} \\
& +\|x(\cdot)\|_{C}\left(L_{0}+\lambda L_{1}\left(\theta-t_{0}\right)+\lambda L_{2} \sqrt{\theta-t_{0}} \frac{1}{c_{1}}\right) \\
= & \beta_{0}+\lambda \beta_{1}\left(\theta-t_{0}\right)+\lambda \beta_{2} \sqrt{\theta-t_{0}} \frac{1}{c_{1}}+L(\lambda)\|x(\cdot)\|_{C}
\end{aligned}
$$

for every $t \in\left[t_{0}, \theta\right]$, and consequently

$$
\|x(\cdot)\|_{C} \leq \beta_{0}+\lambda \beta_{1}\left(\theta-t_{0}\right)+\lambda \beta_{2} \sqrt{\theta-t_{0}} \frac{1}{c_{1}}+L(\lambda)\|x(\cdot)\|_{C} .
$$

Since $L(\lambda)<1$, the last inequality and (4.2) complete the proof.

Proposition 4.2 shows that the set of trajectories $\mathbf{X}$ of the system (3.1) is bounded. 
Now let us prove that the set of trajectories $\mathbf{X}$ of the system (3.1) is a family of equicontinuous functions. Denote

$$
\begin{aligned}
& B_{n}\left(q_{*}\right)=\left\{x \in \mathbb{R}^{n}:\|x\| \leq q_{*}\right\}, \\
& D_{1}=\left[t_{0}, \theta\right] \times B_{n}\left(q_{*}\right), \quad D_{2}=\left[t_{0}, \theta\right] \times\left[t_{0}, \theta\right] \times B_{n}\left(q_{*}\right), \\
& \omega_{0}(\Delta)=\max \left\{\left\|f\left(t_{2}, x\right)-f\left(t_{1}, x\right)\right\|:\left(t_{1}, x\right) \in D_{1},\left(t_{2}, x\right) \in D_{1},\left|t_{2}-t_{1}\right| \leq \Delta\right\}, \\
& \omega_{1}(\Delta)=\max \left\{\left\|K_{1}\left(t_{2}, s, x\right)-K_{1}\left(t_{1}, s, x\right)\right\|:\right. \\
& \\
&\left.\quad\left(t_{1}, s, x\right) \in D_{2},\left(t_{2}, s, x\right) \in D_{2},\left|t_{2}-t_{1}\right| \leq \Delta\right\}, \\
& \omega_{2}(\Delta)=\max \left\{\left\|K_{2}\left(t_{2}, s, x\right)-K_{2}\left(t_{1}, s, x\right)\right\|:\left(t_{1}, s, x\right) \in D_{2},\right. \\
&\left.\left(t_{2}, s, x\right) \in D_{2},\left|t_{2}-t_{1}\right| \leq \Delta\right\}, \\
& \varphi(\Delta)= \frac{1}{1-L_{0}}\left[\omega_{0}(\Delta)+\lambda\left(\theta-t_{0}\right) \omega_{1}(\Delta)+\frac{\lambda \sqrt{\theta-t_{0}}}{c_{1}} \omega_{2}(\Delta)\right] .
\end{aligned}
$$

It is obvious that $\varphi(\cdot):[0, \infty) \rightarrow[0, \infty)$ is not decreasing and $\varphi(\Delta) \rightarrow 0^{+}$as $\Delta \rightarrow 0^{+}$.

Proposition 4.3 For every $t_{1} \in\left[t_{0}, \theta\right], t_{2} \in\left[t_{0}, \theta\right]$, and $x(\cdot) \in \mathbf{X}$ the inequality

$$
\left\|x\left(t_{2}\right)-x\left(t_{1}\right)\right\| \leq \varphi\left(\left|t_{2}-t_{1}\right|\right)
$$

holds, where $\varphi(\cdot)$ is defined by (4.6).

Proof Let $x(\cdot) \in \mathbf{X}$ be an arbitrarily chosen trajectory generated by the admissible control function $u(\cdot) \in U$. Then

$$
x(t)=f(t, x(t))+\lambda \int_{t_{0}}^{\theta}\left[K_{1}(t, s, x(s))+K_{2}(t, s, x(s)) u(s)\right] d s
$$

for every $t \in\left[t_{0}, \theta\right]$, and hence

$$
\begin{aligned}
\left\|x\left(t_{2}\right)-x\left(t_{1}\right)\right\| \leq & \left\|f\left(t_{2}, x\left(t_{2}\right)\right)-f\left(t_{1}, x\left(t_{1}\right)\right)\right\|+\lambda \int_{t_{0}}^{\theta}\left\|K_{1}\left(t_{2}, s, x(s)\right)-K_{1}\left(t_{1}, s, x(s)\right)\right\| d s \\
& +\lambda \int_{a}^{b}\left\|K_{2}\left(t_{2}, s, x(s)\right)-K_{2}\left(t_{1}, s, x(s)\right)\right\|\|u(s)\| d s
\end{aligned}
$$

Since $x(\cdot) \in \mathbf{X}$, according to Proposition 4.2 we have $\|x(s)\| \leq q_{*}$ for every $s \in\left[t_{0}, \theta\right]$. From Condition (3B), (4.3), (4.4), and (4.5) it follows that

$$
\begin{aligned}
& \left\|f\left(t_{2}, x\left(t_{2}\right)\right)-f\left(t_{1}, x\left(t_{1}\right)\right)\right\| \\
& \quad \leq\left\|f\left(t_{2}, x\left(t_{2}\right)\right)-f\left(t_{2}, x\left(t_{1}\right)\right)\right\|+\left\|f\left(t_{2}, x\left(t_{1}\right)\right)-f\left(t_{1}, x\left(t_{1}\right)\right)\right\| \\
& \quad \leq L_{0}\left\|x\left(t_{2}\right)-x\left(t_{1}\right)\right\|+\omega_{0}\left(\left|t_{2}-t_{1}\right|\right), \\
& \left\|K_{1}\left(t_{2}, s, x(s)\right)-K_{1}\left(t_{1}, s, x(s)\right)\right\| \leq \omega_{1}\left(\left|t_{2}-t_{1}\right|\right), \\
& \left\|K_{2}\left(t_{2}, s, x(s)\right)-K_{2}\left(t_{1}, s, x(s)\right)\right\| \leq \omega_{2}\left(\left|t_{2}-t_{1}\right|\right)
\end{aligned}
$$


for any $s \in\left[t_{0}, \theta\right]$. From (2.2), (4.7), (4.8), (4.9), and (4.10) we obtain

$$
\begin{aligned}
\left\|x\left(t_{2}\right)-x\left(t_{1}\right)\right\| \leq & L_{0}\left\|x\left(t_{2}\right)-x\left(t_{1}\right)\right\|+\omega_{0}\left(\left|t_{2}-t_{1}\right|\right)+\lambda\left(\theta-t_{0}\right) \omega_{1}\left(\left|t_{2}-t_{1}\right|\right) \\
& +\lambda \omega_{2}\left(\left|t_{2}-t_{1}\right|\right) \int_{t_{0}}^{\theta}\|u(s)\| d s \\
\leq & L_{0}\left\|x\left(t_{2}\right)-x\left(t_{1}\right)\right\|+\omega_{0}\left(\left|t_{2}-t_{1}\right|\right)+\lambda\left(\theta-t_{0}\right) \omega_{1}\left(\left|t_{2}-t_{1}\right|\right) \\
& +\lambda \omega_{2}\left(\left|t_{2}-t_{1}\right|\right) \cdot \frac{\sqrt{\theta-t_{0}}}{c_{1}} .
\end{aligned}
$$

Since $L_{0} \in[0,1)$, the last inequality and (4.6) imply the proof.

Proposition 4.4 The set of trajectories $\mathbf{X}$ of the system (3.1) is a family of equicontinuous functions.

Proof Let us choose an arbitrary $\varepsilon>0$. Since $\varphi(\Delta) \rightarrow 0^{+}$as $\Delta \rightarrow 0^{+}$, for $\varepsilon>0$ there exists $\delta(\varepsilon)>0$ such that for each $\Delta \in(0, \delta(\varepsilon))$ the inequality $\varphi(\Delta)<\varepsilon$ is satisfied.

Choose an arbitrary $x(\cdot) \in \mathbf{X}$ and $t_{1} \in\left[t_{0}, \theta\right], t_{2} \in\left[t_{0}, \theta\right]$ such that $\left|t_{2}-t_{1}\right|<\delta(\varepsilon)$. Then according to Proposition 4.3 we have

$$
\left\|x\left(t_{2}\right)-x\left(t_{1}\right)\right\| \leq \varphi\left(\left|t_{2}-t_{1}\right|\right)<\varepsilon
$$

and the proof is completed.

Thus from Proposition 4.2, Proposition 4.4, and the Arzela-Ascoli theorem we obtain the precompactness of the set of trajectories.

Theorem 4.1 The set of trajectories $\mathbf{X}$ of the system (3.1) is a precompact subset of the space $C\left(\left[t_{0}, \theta\right], \mathbb{R}^{n}\right)$.

Let $h(E, D)$ denote the Hausdorff distance between the sets $E \subset \mathbb{R}^{n}$ and $D \subset \mathbb{R}^{n}$. From Proposition 4.3 follows the validity of the following corollary.

Corollary 4.1 For every $t_{1} \in\left[t_{0}, \theta\right]$ and $t_{2} \in\left[t_{0}, \theta\right]$ the inequality

$$
h\left(\mathbf{X}\left(t_{1}\right), \mathbf{X}\left(t_{2}\right)\right) \leq \varphi\left(\left|t_{2}-t_{1}\right|\right)
$$

is satisfied, and hence the set valued map $t \rightarrow \mathbf{X}(t), t \in\left[t_{0}, \theta\right]$, is continuous in the Hausdorff metric, where the set $\mathbf{X}(t)$ is defined by (3.3).

\section{Closedness of the set of trajectories}

The next theorem specifies closedness of the set of trajectories $\mathbf{X}$ of the system (3.1).

Proposition 5.1 The set of trajectories $\mathbf{X}$ of the system (3.1) is a closed subset of the space $C\left(\left[t_{0}, \theta\right] ; \mathbb{R}^{n}\right)$.

Proof Suppose that $x_{k}(\cdot) \in \mathbf{X}$ for every $k=1,2, \ldots$ and $\left\|x_{k}(\cdot)-x_{0}(\cdot)\right\|_{C} \rightarrow 0$ as $k \rightarrow \infty$. Let the trajectory $x_{k}(\cdot)$ be generated by the admissible control function $u_{k}(\cdot) \in U$, where $k=$ $1,2, \ldots$ According to Proposition 2.3, the set of admissible control functions $U$ is weakly 
compact in the space $L_{2}\left(\left[t_{0}, \theta\right] ; \mathbb{R}^{m}\right)$. Then, without loss of generality, one can assume that $u_{k}(\cdot) \stackrel{\text { weak }}{\longrightarrow} u_{*}(\cdot)$ as $k \rightarrow \infty$, where $u_{*}(\cdot) \in U$. Let $x_{*}(\cdot):\left[t_{0}, \theta\right] \rightarrow \mathbb{R}^{n}$ be the trajectory of system (3.1), generated by $u_{*}(\cdot) \in U$. Then $x_{*}(\cdot) \in \mathbf{X}$ and via condition (3B) we have

$$
\begin{aligned}
\left\|x_{k}(t)-x_{*}(t)\right\| \leq & \left\|f\left(t, x_{k}(t)\right)-f\left(t, x_{*}(t)\right)\right\|+\lambda \int_{t_{0}}^{\theta}\left\|K_{1}\left(t, s, x_{k}(s)\right)-K_{1}\left(t, s, x_{*}(s)\right)\right\| d s \\
& +\lambda\left\|\int_{t_{0}}^{\theta}\left[K_{2}\left(t, s, x_{k}(s)\right) u_{k}(s)-K_{2}\left(t, s, x_{*}(s)\right) u_{*}(s)\right] d s\right\| \\
\leq & L_{0}\left\|x_{k}(s)-x_{*}(s)\right\|+\lambda L_{1} \int_{t_{0}}^{\theta}\left\|x_{k}(s)-x_{*}(s)\right\| d s \\
& +\lambda\left\|\int_{t_{0}}^{\theta}\left[K_{2}\left(t, s, x_{k}(s)\right) u_{k}(s)-K_{2}\left(t, s, x_{*}(s)\right) u_{*}(s)\right] d s\right\|
\end{aligned}
$$

for any $t \in\left[t_{0}, \theta\right]$. Since $L_{0} \in[0,1)$, the last inequality yields

$$
\begin{aligned}
\left\|x_{k}(t)-x_{*}(t)\right\| \leq & \frac{\lambda L_{1}}{1-L_{0}} \int_{t_{0}}^{\theta}\left\|x_{k}(s)-x_{*}(s)\right\| d s \\
& +\frac{\lambda}{1-L_{0}}\left\|\int_{t_{0}}^{\theta}\left[K_{2}\left(t, s, x_{k}(s)\right) u_{k}(s)-K_{2}\left(t, s, x_{*}(s)\right) u_{*}(s)\right] d s\right\|
\end{aligned}
$$

for every $t \in\left[t_{0}, \theta\right]$.

Condition (3B) implies that

$$
\begin{aligned}
& \left\|\int_{t_{0}}^{\theta}\left[K_{2}\left(t, s, x_{k}(s)\right) u_{k}(s)-K_{2}\left(t, s, x_{*}(s)\right) u_{*}(s)\right] d s\right\| \\
& \quad \leq \int_{t_{0}}^{\theta} L_{2}\left\|x_{k}(s)-x_{*}(s)\right\|\left\|u_{k}(s)\right\| d s+\left\|\int_{t_{0}}^{\theta} K_{2}\left(t, s, x_{*}(s)\right)\left(u_{k}(s)-u_{*}(s)\right) d s\right\| .
\end{aligned}
$$

Setting $\psi(t, s)=K_{2}\left(t, s, x_{*}(s)\right)$, from (5.1) and (5.2) we obtain

$$
\begin{aligned}
\left\|x_{k}(t)-x_{*}(t)\right\| \leq & \frac{\lambda}{1-L_{0}} \int_{t_{0}}^{\theta}\left(L_{1}+L_{2}\left\|u_{k}(s)\right\|\right)\left\|x_{k}(s)-x_{*}(s)\right\| d s \\
& +\frac{\lambda}{1-L_{0}}\left\|\int_{t_{0}}^{\theta} \psi(t, s)\left(u_{k}(s)-u_{*}(s)\right) d s\right\|
\end{aligned}
$$

for every $t \in\left[t_{0}, \theta\right]$.

Since the function $\psi(\cdot, \cdot):\left[t_{0}, \theta\right] \times\left[t_{0}, \theta\right] \rightarrow \mathbb{R}^{n \times m}$ is continuous, $u_{k}(\cdot) \stackrel{\text { weak }}{\longrightarrow} u_{*}(\cdot)$ as $k \rightarrow \infty$, for each fixed $t \in\left[t_{0}, \theta\right]$ we have

$$
\int_{t_{0}}^{\theta} \psi(t, s)\left(u_{k}(s)-u_{*}(s)\right) d s \rightarrow 0 \quad \text { as } k \rightarrow \infty .
$$

Thus, for a fixed $t \in\left[t_{0}, \theta\right]$ and for a given $\varepsilon>0$ there exists $K_{*}(t, \varepsilon)>0$ such that for each $k>K_{*}(t, \varepsilon)$ the inequality

$$
\left\|\int_{t_{0}}^{\theta} \psi(t, s)\left(u_{k}(s)-u_{*}(s)\right) d s\right\|<\varepsilon
$$

is satisfied. 
Now let us prove that for a given $\varepsilon>0$, there exists $K^{*}(\varepsilon)>0$ such that for each $k>K^{*}(\varepsilon)$ and $t \in\left[t_{0}, \theta\right]$ the inequality

$$
\left\|\int_{t_{0}}^{\theta} \psi(t, s)\left(u_{k}(s)-u_{*}(s)\right) d s\right\|<\varepsilon
$$

holds.

Assume the contrary. Then there exist $\varepsilon_{*}>0, t_{i} \in\left[t_{0}, \theta\right], k_{i}>0$ such that $k_{i} \rightarrow+\infty$ as $i \rightarrow+\infty$ and the inequality

$$
\left\|\int_{t_{0}}^{\theta} \psi\left(t_{i}, s\right)\left(u_{k_{i}}(s)-u_{*}(s)\right) d s\right\| \geq \varepsilon_{*}
$$

is verified for every $i=1,2, \ldots$.

Since $t_{i} \in\left[t_{0}, \theta\right]$ for each $i=1,2, \ldots$, without loss of generality, assume that $t_{i} \rightarrow t_{*} \in$ $\left[t_{0}, \theta\right]$ as $i \rightarrow+\infty$.

According to (5.4), for $\frac{\varepsilon_{*}}{4}>0$ there exists $K_{1}\left(t_{*}, \varepsilon_{*}\right)>0$ such that for each $i>K_{1}\left(t_{*}, \varepsilon_{*}\right)$ the inequality

$$
\left\|\int_{t_{0}}^{\theta} \psi\left(t_{*}, s\right)\left(u_{k_{i}}(s)-u_{*}(s)\right) d s\right\|<\frac{\varepsilon_{*}}{4}
$$

holds.

The continuity of the function $\psi(\cdot, \cdot):\left[t_{0}, \theta\right] \times\left[t_{0}, \theta\right] \rightarrow \mathbb{R}^{n \times m}$ shows that for $\frac{\varepsilon_{*} c_{1}}{8 \sqrt{\theta-t_{0}}}$ there exists $K_{2}\left(t_{*}, \varepsilon_{*}\right)$ such that for every $i>K_{2}\left(t_{*}, \varepsilon_{*}\right)$ and $s \in\left[t_{0}, \theta\right]$ the inequality

$$
\left\|\psi\left(t_{i}, s\right)-\psi\left(t_{*}, s\right)\right\|<\frac{\varepsilon_{*} c_{1}}{8 \sqrt{\theta-t_{0}}}
$$

is satisfied.

Denote $K_{3}\left(t_{*}, \varepsilon_{*}\right)=\max \left\{K_{1}\left(t_{*}, \varepsilon_{*}\right), K_{2}\left(t_{*}, \varepsilon_{*}\right)\right\}$. Since $u_{k_{i}}(\cdot) \in U, u_{*}(\cdot) \in U,(2.2),(5.7)$, and (5.8) yield, for every $i>K_{3}\left(t_{*}, \varepsilon_{*}\right)$, the inequality

$$
\begin{aligned}
\left\|\int_{t_{0}}^{\theta} \psi\left(t_{i}, s\right)\left(u_{k_{i}}(s)-u_{*}(s)\right) d s\right\| \leq & \left\|\int_{t_{0}}^{\theta}\left[\psi\left(t_{i}, s\right)-\psi\left(t_{*}, s\right)\right]\left(u_{k_{i}}(s)-u_{*}(s)\right) d s\right\| \\
& +\left\|\int_{t_{0}}^{\theta} \psi\left(t_{*}, s\right)\left(u_{k_{i}}(s)-u_{*}(s)\right) d s\right\| \\
& <\frac{\varepsilon_{*}}{4}+\int_{t_{0}}^{\theta}\left\|\psi\left(t_{i}, s\right)-\psi\left(t_{*}, s\right)\right\|\left\|u_{k_{i}}(s)-u_{*}(s)\right\| d s \\
\leq & \frac{\varepsilon_{*}}{4}+\int_{t_{0}}^{\theta} \frac{\varepsilon_{*} c_{1}}{8 \sqrt{\theta-t_{0}}}\left[\left\|u_{k_{i}}(s)\right\|+\left\|u_{*}(s)\right\|\right] d s \\
\leq & \frac{\varepsilon_{*}}{4}+\frac{\varepsilon_{*} c_{1}}{8 \sqrt{\theta-t_{0}}} \cdot \frac{2 \sqrt{\theta-t_{0}}}{c_{1}}=\frac{\varepsilon_{*}}{2} .
\end{aligned}
$$

Thus (5.6) and (5.9) are in contradiction, and hence the validity of (5.5) is proved. 
Now, for a given $\varepsilon>0$, let us choose an arbitrary $k>K^{*}(\varepsilon)$. Then from (2.2), (3.2), (5.3), and (5.5) it follows that

$$
\begin{aligned}
\left\|x_{k}(t)-x_{*}(t)\right\| & \leq \frac{\lambda}{1-L_{0}} \varepsilon+\frac{\lambda}{1-L_{0}} \int_{t_{0}}^{\theta}\left(L_{1}+L_{2}\left\|u_{k}(s)\right\|\right)\left\|x_{k}(s)-x_{*}(s)\right\| d s \\
& \leq \frac{\lambda}{1-L_{0}} \varepsilon+\frac{\lambda}{1-L_{0}}\left\|x_{k}(\cdot)-x_{*}(\cdot)\right\|_{C} \int_{t_{0}}^{\theta}\left(L_{1}+L_{2}\left\|u_{k}(s)\right\|\right) d s \\
& =\frac{\lambda}{1-L_{0}} \varepsilon+\frac{\lambda}{1-L_{0}}\left(L_{1}\left(\theta-t_{0}\right)+L_{2} \int_{t_{0}}^{\theta}\left\|u_{k}(s)\right\| d s\right)\left\|x_{k}(\cdot)-x_{*}(\cdot)\right\|_{C} \\
& \leq \varepsilon \frac{\lambda}{1-L_{0}}+\frac{\lambda}{1-L_{0}}\left(L_{1}\left(\theta-t_{0}\right)+L_{2} \frac{\sqrt{\theta-t_{0}}}{c_{1}}\right)\left\|x_{k}(\cdot)-x_{*}(\cdot)\right\|_{C} \\
& =\varepsilon \frac{\lambda}{1-L_{0}}+\frac{L(\lambda)-L_{0}}{1-L_{0}}\left\|x_{k}(\cdot)-x_{*}(\cdot)\right\|_{C}
\end{aligned}
$$

for every $t \in\left[t_{0}, \theta\right]$, and consequently

$$
\left\|x_{k}(\cdot)-x_{*}(\cdot)\right\|_{C} \leq \frac{\lambda}{1-L_{0}} \varepsilon+\frac{L(\lambda)-L_{0}}{1-L_{0}}\left\|x_{k}(\cdot)-x_{*}(\cdot)\right\|_{C}
$$

for any $k>K_{*}(\varepsilon)$. Since

$$
1-\frac{L(\lambda)-L_{0}}{1-L_{0}}>0
$$

(5.10) implies that

$$
\left\|x_{k}(\cdot)-x_{*}(\cdot)\right\|_{C} \leq \frac{\lambda}{1-L(\lambda)} \cdot \varepsilon
$$

for any $k>K^{*}(\varepsilon)$. This means that $\left\|x_{k}(\cdot)-x_{*}(\cdot)\right\|_{C} \rightarrow 0$ as $k \rightarrow \infty$. The uniqueness of limit gives us that $x_{0}(\cdot)=x_{*}(\cdot)$. Since $x_{*}(\cdot) \in \mathbf{X}, x_{0}(\cdot) \in \mathbf{X}$ and the proof is completed.

Theorem 4.1 and Theorem 5.1 yield the compactness of the set of trajectories.

Theorem 5.1 The set of trajectories $\mathbf{X}$ of the system (3.1) is a compact subset of the space $C\left(\left[t_{0}, \theta\right] ; \mathbb{R}^{n}\right)$.

\section{Conclusion}

Compactness of the set of trajectories of the control system described by a Urysohn type integral equation is specified where the system is nonlinear with respect to the state vector and is affine with respect to the control vector. The admissible control functions are chosen from the space $L_{2}\left(\left[t_{0}, \theta\right] ; \mathbb{R}^{m}\right)$ which satisfy an additional quadratic integral constraint. This means that the control resource of the system is limited and it is exhausted by consumption. It is proved that the set of trajectories is a compact subset of the space $C\left(\left[t_{0}, \theta\right] ; \mathbb{R}^{n}\right)$. This allows one to predict the existence of the optimal trajectory in the optimal control problem for the system described by a Urysohn type integral equation with quadratic integral constraint on the controls and with continuous payoff functional. 


\section{Competing interests}

The authors declare that they have no competing interests.

\section{Authors' contributions}

All authors read and approved the final manuscript.

\section{Author details}

${ }^{1}$ Department of Mathematics, Faculty of Science, Universiti Putra Malaysia, Serdang, Selangor 43400, Malaysia. ${ }^{2}$ Faculty of Education, Cumhuriyet University, Sivas, 58140, Turkey. ${ }^{3}$ Faculty of Science, Cumhuriyet University, Sivas, 58140, Turkey.

\section{Acknowledgements}

This work has been fully supported by Universiti Putra Malaysia Research Fund.

Received: 25 June 2015 Accepted: 24 November 2015 Published online: 01 February 2016

\section{References}

1. Agarwal, RP, Banas, J, Banas, K, O'Regan, D: Solvability of a quadratic Hammerstein integral equation in the class of functions having limits at infinity. J. Integral Equ. Appl. 23, 157-181 (2011)

2. Appell, J, De Pascale, E, Zabreyko, PP: On the application of the Newton-Kantorovich method to nonlinear integral equations of Uryson type. Numer. Funct. Anal. Optim. 12, 271-283 (1991)

3. Banas, J, O'Regan, D, Agarwal, RP: Measures of noncompactness and asymptotic stability of solutions of a quadratic Hammerstein integral equation. Rocky Mt. J. Math. 41, 1769-1792 (2011)

4. Brauer, F: On a nonlinear integral equation for population growth problems. SIAM J. Math. Anal. 6, $312-317$ (1975)

5. Browder, FE: Nonlinear functional analysis and nonlinear integral equations of Hammerstein and Urysohn type. In: Contributions to Nonlinear Functional Analysis. Proc. Sympos., Math. Res. Center, Univ. Wisconsin, pp. 425-500. Academic Press, New York (1971)

6. Burton, TA: Six integral equations and a flexible Lyapunov functional. In: Proc. Inst. Math. Mech. Ural Branch of Russian Acad. Sci., vol. 16, pp. 241-252 (2010)

7. Hochstadt, H: Integral Equations. Wiley, New York (1973)

8. Krasnoselskii, MA, Krein, SG: On the principle of averaging in nonlinear mechanics. Usp. Mat. Nauk 10, 147-153 (1955) (in Russian)

9. Minorsky, N: Introduction to Nonlinear Mechanics. Edwards, Ann Arbor (1947)

10. Polyanin, AD, Manzhirov, AV: Handbook of Integral Equation. CRC Press, Boca Raton (1998)

11. Precup, R: Methods in Nonlinear Integral Equations. Kluwer Academic, Dordrecht (2002)

12. Urysohn, PS: On a type of nonlinear integral equation. Mat. Sb. 31(2), 236-255 (1923) (in Russian)

13. Angell, TS, George, RK, Sharma, JP: Controllability of Urysohn integral inclusions of Volterra type. Electron. J. Differ. Equ. 2010, 79 (2010)

14. Balder, EJ: On existence problems for the optimal control of certain nonlinear integral equations of Urysohn type. J. Optim. Theory Appl. 42(3), 447-465 (1984)

15. Bennati, ML: An existence theorem for optimal controls of systems defined by Uryson integral equations. Ann. Mat Pura Appl. 4, 187-197 (1979) (in Italian)

16. Chentsov, AG: Approximative realization of integral constraints and generalized constructions in the class of vector finitely additive measures. Proc. Steklov Inst. Math. Suppl. 2, S10-S60 (2002)

17. Conti, R: Problemi di Controllo e di Controllo Ottimale. UTET, Torino (1974)

18. Guseinov, KG, Neznakhin, AA, Ushakov, VN: Approximate construction of reachable sets of control systems with integral constraints on the controls. J. Appl. Math. Mech. 63(4), 557-567 (1999)

19. Krasovskii, NN: Theory of Control of Motion: Linear Systems. Nauka, Moscow (1968) (in Russian)

20. Subbotin, Al, Subbotina, NN: Alternative for the encounter-evasion differential game with constraints on the momenta of the players controls. J. Appl. Math. Mech. 39, 376-385 (1975)

21. Subbotin, Al, Ushakov, VN: Alternative for an encounter-evasion differential game with integral constraints on the players controls. J. Appl. Math. Mech. 39, 367-375 (1979)

22. Vdovina, OI, Sesekin, AN: Numerical construction of attainability domains for systems with impulse control. Proc. Steklov Inst. Math. Suppl. 1, S246-S255 (2005)

23. Ukhobotov, Vl: One Dimensional Projection Method in Linear Differential Games with Integral Constraints. Chelyabinsk University Press, Chelyabinsk (2005) (in Russian)

24. Huseyin, N, Huseyin, A: Compactness of the set of trajectories of the controllable system described by an affine integral equation. Appl. Math. Comput. 219, 8416-8424 (2013)

25. Huseyin, A, Huseyin, N: Dependence on the parameters of the set of trajectories of the control system described by a nonlinear Volterra integral equation. Appl. Math. 59(3), 303-317 (2014)

26. Huseyin, A, Huseyin, N: Precompactness of the set of trajectories of the controllable system described by a nonlinear Volterra integral equation. Math. Model. Anal. 17(5), 686-695 (2012)

27. Aubin, J-P: Optima and Equilibria. An Introduction to Nonlinear Analysis. Springer, Berlin (1998)

28. Riesz, F, Sz.-Nagy, B: Functional Analysis. Dover, New York (1990)

29. Yosida, K: Functional Analysis. Springer, New York (1980) 